\title{
Depletion of juvenile hormone esterase extends larval growth in Bombyx mori
}

Zhongjie Zhang ${ }^{\mathrm{a}, \mathrm{b}}$, Xiaojing Liu ${ }^{\mathrm{b}}$, Takahiro Shiotsuki ${ }^{\mathrm{c}}$, Zhisheng Wang ${ }^{\mathrm{d}}$, Xia Xu $\mathrm{Xu}^{\mathrm{a}}$, Yongping Huang ${ }^{\mathrm{b}}$, Muwang $\mathrm{Li}^{\mathrm{e}}$, Kai Li ${ }^{\mathrm{a}}$,, Anjiang Tan ${ }^{\mathrm{b}, ~ *}$

a School of Life Science, East China Normal University, Shanghai 200062, China

b Institute of Plant Physiology and Ecology, Shanghai Institutes for Biological Sciences, Chinese Academy of Sciences, Shanghai 200032, China

c Insect Growth Regulation Research Unit, National Institute of Agrobiological Sciences, Tsukuba, Ibaraki, Japan

d College of Fisheries and Life Science, Shanghai Ocean University, Shanghai 201306, China

e Sericultural Research Institute, Jiangsu University of Science and Technology, Zhenjiang 212018, Jiangsu, China

Correspondence to:

Kai Li, E-mail: kaili@bio.ecnu.edu.cn, Tel: +86-021-54345461; Anjiang Tan, E-mail: ajtan01@sibs.ac.cn,Tel: +86-21-54924046. 


\section{Abstract}

Two major hormones, juvenile hormone (JH) and 20-hydroxyecdysone (20E), regulate insect growth and development according to their precisely coordinated titres, which are controlled by both biosynthesis and degradation pathways. Juvenile hormone esterase (JHE) is the primary $\mathrm{JH}$-specific degradation enzyme that plays a key role in regulating JH titers, along with $\mathrm{JH}$ epoxide hydrolase (JHEH) and JH diol kinase (JHDK). In the current study, a loss-of-function analysis of JHE in the silkworm, Bombyx mori, was performed by targeted gene disruption using the transgenic CRISPR/Cas9 (clustered regularly interspaced short palindromic repeats/RNA-guided Cas9 nucleases) system. Depletion of B. mori JHE (BmJHE) resulted in the extension of larval stages, especially the penultimate and ultimate larval stages, without deleterious effects to silkworm physiology. The expression of JHEH and JHDK was upregulated in mutant animals, indicating the existence of complementary routes in the $\mathrm{JH}$ metabolism pathway in which inactivation of one enzyme will activate other enzymes. RNA-Seq analysis of mutant animals revealed that genes involved in protein processing in the endoplasmic reticulum and in amino acid metabolism were affected by BmJHE depletion. Depletion of JHE and subsequent delayed JH metabolism activated genes in the TOR pathway, which are ultimately responsible for extending larval growth. The transgenic Cas 9 system used in the current study provides a promising approach for analysing the actions of $\mathrm{JH}$, especially in nondrosophilid insects. Furthermore, prolonging larval stages produced larger larvae and cocoons, which is greatly beneficial to silk production.

Key words: Bombyx mori, juvenile hormone esterase, CRISPR/Cas9 


\section{Introduction}

Insect growth and development are orchestrated by ecdysteroids and juvenile hormones (JHs) (Gäde et al., 1997; Jindra et al., 2013). Ecdysteroids are produced in the prothoracic glands and initiate and orchestrate the moulting process (Niwa et al., 2014). JHs are produced in the corpora allata (CA) and modulate the action of ecdysteroids and prevent premature metamorphosis (Riddiford et al., 1996). In the presence of JHs, ecdysteroids ensure a moult to the next larval stage, whereas the larva undergoes metamorphosis in the absence of JHs (Riddiford et al., 2010). Thus, the precise regulation of both hormone titres is critical in the process of insect moulting and metamorphosis.

JHs belong to the family of sesquiterpenes and play key roles in a wide spectrum of insect biological events, including development and metamorphosis (Riddiford et al., 2010), reproduction (Bellés et al., 2005) and polyphenisms (Truman and Riddiford, 2007). JHs are synthesized in the corpora allata, released into the haemolymph and transported to target tissues to regulate corresponding physiological processes (Riddiford et al., 2012). Comparing numerous genes have been identified in regulating JH biosynthesis (Bellés et al., 2005), only three primary JH metabolic enzymes, i.e., JH esterase (JHE), JH epoxide hydrolase (JHEH) and JH diol kinase (JHDK), have been identified (Hirai et al., 2002; Li et al., 2005; Seino et al., 2010). Among them, JHE is the most extensively investigated gene which has been proved to play key roles in JH metabolism pathway (Kamita and Hammock, 2010).

Altering the $\mathrm{JH}$ titre in vivo can be achieved by regulating JHE activity. In a previous study, we demonstrated that the transgenic overexpression of JHE induced precocious metamorphosis in the lepidopteran model insect Bombyx mori (Tan et al., 2005). By contrast, the inhibition of JHE activity by a chemical inhibitor, O-ethyl-S-phenyl phosphoramidothiolate, extends the feeding stage and delays pupation in Trichoplusia ni (Sparks and Hammock, 1980). However, the application of chemical inhibitors may have unpredictably deleterious effects on insect physiology, thus limiting its potential application in the growth control of beneficial 
insects. RNA interference (RNAi) is an efficient genetic tool for knocking down target gene expression in insects. However, in vivo use of RNAi is still challenging due to its inefficiency in most lepidopteran insects including $B$.mori. The recent emergence of genome-editing tools such as ZFN (zinc-finger nucleases), TALEN (transcription-activator like effector nucleases) and the CRISPR/Cas9 (clustered regularly interspaced short palindromic repeats/RNA-guided Cas9 nucleases) systems provide robust loss-of-function toolkits In the lepidopteran model insect B. mori, these techniques have been established, including the transgenic TALEN and Cas9 systems ( $\mathrm{Xu}$ et al., 2014; $\mathrm{Li}$ et al., 2015). Although the transgenic CRISPR/Cas9-mediated gene knockout could not result in complete gene depletion, this somatic mutagenesis system provides an efficient alternative to perform gene loss-of-function analysis in those insect species where RNAi is inefficient.

In the current study, we produced somatic mutagenesis in BmJHE loci by using the transgenic CRISPR/Cas9 system. Depletion of BmJHE decreased JHE activity, which resulted in the extension of growth during the late larval stages (fourth and fifth instars) but not in the early stages (first to third instars). RNA-seq and subsequent q-RT-PCR analyses suggested that life spans mediated by JH were associated with the insulin/TOR pathway. Furthermore, the cocoon yield of JHE-mutant animals increased significantly due to the extension of the larval period, which will greatly contribute to silk production.

\section{Materials and Methods}

\subsection{Silkworm strains}

A multivoltine silkworm strain, Nistari, was used for all experiments. Larvae were reared on fresh mulberry leaves at $25^{\circ} \mathrm{C}$ under standard conditions (Tan et al., 2013).

\subsection{Plasmid construction}

To construct the transgenic CRISPR/Cas9 system, we used the activator line of 
pBac[3xp3-DsRed2-IE1-Cas9] (IE1-Cas9), in which Cas9 was driven by an ubiquitous IE1 promoter, as described previously (Li et al., 2015). To construct the effector line pBac[IE1-EGFP-U6-BmJHEgRNAs] (U6-sgRNAs), the plasmid pBac[IE1-EGFP] was used as the initial plasmid. The first U6 promoter sequences were PCR-amplified from silkworm genomic DNA using the primers HR-F and U6-R1. A primer pair, $s g R N A-\mathrm{F} 1$ and Overlap-R, was annealed to generate BmJHE sgRNA1. The second U6 promoter sequence was also PCR-amplified from silkworm genomic DNA using the primers Overlap-F and U6-R2. $S g R N A-\mathrm{F} 2$ and HR-R were annealed to generate BmJHE sgRNA2. Overlap extension PCR was performed with 1 $\mu \mathrm{l}$ of $50 \mathrm{ng} / \mu \mathrm{l}$ of the first $U 6$ promoter product and $1 \mu \mathrm{l}$ of $10 \mathrm{ng} / \mu \mathrm{BmJHE} \operatorname{sgRNA} 1$ template in a $50 \mu \mathrm{l}$ PCR reaction mix. Subsequently, the products of the overlap extension PCR were sub-cloned into pBac[IE1-EGFP] using the ClonExpress MultiS One Step Cloning Kit (Vazyme, Nanjing, China). The primers used for plasmid construction are listed in Supplementary Material: Table S1.

\subsection{Silkworm germline transformation}

For silkworm germline transformation, preblastoderm Nistari embryos were injected with a mixture of transformation plasmids and helper plasmids and subsequently incubated at $25^{\circ} \mathrm{C}$ in a humidified chamber for 10-12 days until larval hatching (Tan et al., 2005). Hatched larvae were reared to moths and sib-mated or backcrossed with wild-type moths. G1 progeny were scored for the presence of the marker gene during the embryonic stage under a fluorescence microscope (Nikon AZ100, Japan). For the transgenic CRISPR/Cas9 system, the IE1-Cas9 line was crossed with the U6-BmJHE sgRNA line, and the heterozygous F1 progeny were used in subsequent experiments.

\subsection{RNA isolation and cDNA synthesis}

Total RNA was isolated from the fat body on the third day of the fifth larval instar (L5D3) using TRIzol reagent (Invitrogen, CA, USA) according to the manufacturer's 
instructions and subsequently was treated with DNase I (Invitrogen, CA, USA) to remove genomic DNA. To synthesize cDNA, $1 \mu \mathrm{g}$ of total RNA was used using the ReverAid First Strand cDNA Synthesis Kit (Fermentas, Lithuania, EU).

\subsection{Quantitative real-time PCR ( $q-R T-P C R)$ analysis}

Q-RT-PCR was performed to analyse the expression levels of selected genes after BmJHE depletion. cDNA synthesized from total RNA isolated from the fat body at L5D3 was used as the template. The PCR conditions were as follows: initial incubation at $95^{\circ} \mathrm{C}$ for $1 \mathrm{~min}, 40$ cycles of $95^{\circ} \mathrm{C}$ for $15 \mathrm{sec}$, and $60^{\circ} \mathrm{C}$ for $1 \mathrm{~min}$. The cycling conditions were as follows: initial incubation at $95^{\circ} \mathrm{C}$ for $10 \mathrm{~min}, 45$ cycles of $95^{\circ} \mathrm{C}$ for $15 \mathrm{sec}$, and $60^{\circ} \mathrm{C}$ for $1 \mathrm{~min}$. The primers that were used in q-RT-PCR to investigate the mRNA expression levels of the genes of interest are listed in Supplementary Material: Table S1. Another primer pair, RP49-F and RP49-R (Table S1), which amplifies a 136-bp fragment from the B. mori ribosomal protein 49, was used as an internal control (Tan et al., 2013).

\subsection{Genomic DNA extraction and mutagenesis analysis}

Genomic PCR, followed by sequencing, was carried out to identify the BmJHE mutant alleles induced by the transgenic CRISPR/Cas9 system. Genomic DNA was extracted from the heterozygote with the DNA extraction buffer $(1: 1: 2: 2.5$ ratio of $10 \%$ SDS to $5 \mathrm{M} \mathrm{NaCl}$ to $100 \mathrm{mM}$ EDTA to $500 \mathrm{mM}$ Tris- $\mathrm{HCl}, \mathrm{pH}=8$ ), incubated with proteinase $\mathrm{K}$ and purified via a standard phenol:chloroform extraction and isopropanol precipitation extraction, followed by RNaseA treatment. The PCR conditions were as follows: $98^{\circ} \mathrm{C}$ for $2 \mathrm{~min}, 35$ cycles of $94^{\circ} \mathrm{C}$ for $10 \mathrm{~s}, 55^{\circ} \mathrm{C}$ for $30 \mathrm{~s}$, and $72^{\circ} \mathrm{C}$ for $40 \mathrm{~s}$, followed by a final extension period of $72^{\circ} \mathrm{C}$ for $10 \mathrm{~min}$. The PCR products were cloned into pJET1.2 vectors (Fermentas, Lithuania, EU) and directly sequenced. The primers that were designed to detect mutagenesis in targeted sites were as follows: JHE-TS-F and JHE-TS-R detected targeting sites. The primer sequences are listed in Supplementary Material: Table S1. 


\subsection{Inverse PCR}

Inverse PCR was performed as previously described (Tan et al., 2013) to locate the insertion loci of transgenic silkworm lines. The genomic DNA was extracted from G1 transgenic moths and incubated with proteinase $\mathrm{K}$ and then purified by standard SDS lysis-phenol extraction treatment, followed by RNaseA treatment. DNA of transgenic lines was digested with $B f u C I$ (NEB, Ipswich, MA) and circularized by T4 ligase (Thermo Fisher Scientific, Waltham, MA) overnight at $16{ }^{\circ} \mathrm{C}$. PCR was performed using the circularized fragments as templates with primers designed from the $5^{\prime}$ arm of the piggyBac vector: 5'-F1 and 5'-R1 for the first PCR and 5'-F2 and 5'-R2 for the second PCR (Table S1). The PCR conditions were as follows: $94{ }^{\circ} \mathrm{C}$ for $1 \mathrm{~min}, 35$ cycles of $94{ }^{\circ} \mathrm{C}$ for $30 \mathrm{~s}, 55{ }^{\circ} \mathrm{C}$ for $30 \mathrm{~s}$, and $68{ }^{\circ} \mathrm{C}$ for $2 \mathrm{~min}$, followed by a final extension period of $68{ }^{\circ} \mathrm{C}$ for $5 \mathrm{~min}$. Amplified products were gel-purified and sequenced directly.

\subsection{Determination of JHE activity}

JHE activity in the larval haemolymph was determined using a spectrophotometric assay with methyl 1-heptylthioacetothioate as a JH-mimic substrate (McCutchen et al., 1995). Absorbance at $405 \mathrm{~nm}$ was recorded on a microplate reader (Spectra-Max, Molecular Devices, Sunnyvale, USA) for $5 \mathrm{~min}$. The enzymatic activity was determined in triplicate.

\subsection{RNA-seq analysis}

A total of $20 \mu \mathrm{g}$ of the RNA samples extracted from the fat body dissected from U6-sgRNAs and IE1-Cas9:U6-sgRNAs line was used to generate RNA-seq libraries using the Illumina TruSeq ${ }^{\mathrm{TM}}$ RNA Sample Preparation kit (Illumina, CA, USA) following the manufacturer's recommendations. Total mRNA was isolated using Sera-mag Magnetic Oligo (dT) Beads (Illumina, CA, USA). The mRNA was fragmented (200 nt) and used for cDNA synthesis. Sequencing adaptors were ligated to cDNA by PCR amplification. The cDNA libraries were sequenced using the 
Illumina HiSeq 2000 platform (BGI, Wuhan, China). Sequenced raw data were qualified, filtered and mapped to the reference silkworm genome database (http://www.silkdb.org/silkdb/) by tophat/bowtie2. Unigene abundances were measured by fragment per kilobase of exon per million fragments mapped (FPKM). To analysis the differentially expressed genes (DEGs), NOIseq method was used to identify DEGs with cutoff P-value $<0.001$ (Tarazona et al., 2011). For further analysis, we applied the Blast2GO (Conesa et al., 2005) software to get gene ontology (GO) annotation of DEGs defined by molecular function, cellular component and biological process ontologies. After getting GO annotation for DEGs, we used WEGO software (Kanehisa et al., 2008) to do GO functional classification for DEGs and to understand the distribution of gene functions of the species from the macro level. Pathway assignments were determined following the Kyoto Encyclopedia of Genes and Genomes (KEGG) database using tBLASTn with an E-value $<1$ e-5.

\section{Results}

\subsection{Plasmid construction and silkworm germline transformation.}

The BmJHE locus is located on chromosome 25 and its coding region is composed of six exons and five introns (Fig. 1a). The open reading frame of BmJHE was screened to identify sgRNA targeting sites according to the GGN19GG rule (Hwang et al., 2013). Two 23-bp sgRNA target sites, GGAAGTCTGATGAAACCTCACGG and GGTGAGACGCCTACGAAGCCTGG, were identified in exon three and named S1 and S2, respectively (Fig. 1a). The transgenic vector pBac[U6-BmJHEsgRNAs-IE1-DsRed2] was constructed to contain these two U6 sgRNA expression cassettes with the EGFP fluorescent marker (Fig. 1b). Another transgenic vector, $p B a c[3 x p 3-E G F P-I E 1-C a s 9]$, was constructed to contain a Cas9 open reading frame $(\mathrm{ORF})$ driven by a ubiquitous IE1 promoter with the EGFP fluorescent marker as described previously (Fig. 1c, Li et al., 2015).

Overall, 480 preblastoderm Nistari embryos (G0) were microinjected with a 
mixture of U6-sgRNAs and helper plasmids. Among them, 316 eggs $(65 \%)$ hatched, and 127 larvae survived to the adult stage. These G0 moths were sib-mated or mated to wild-type (WT) moths, generating 58 broods (G1). Using a fluorescence microscope (Nikon AZ100), we detected nine broods with GFP-positive individuals. Three distinct transgenic lines, B1, B2 and B3, were used in the subsequent experiments. The insertion of the transgenes into the genome was confirmed by inverse-PCR. The transgene in the B1-3 line was localized in chromosome 10 between two undefined genes, KAIKOGA022440 and KAIKOGA022441, chromosome 11 between two undefined genes, KAIKOGA033853 and KAIKOGA033852, and chromosome 13 between two exons of KAIKOGA003726 (Fig. S1).

\subsection{Depletion of BmJHE using the CRISPR/Cas9 system}

The binary transgenic TALEN and the CRISPR/Cas9 systems in B. mori were recently established in our laboratory to perform a somatic loss-of-function analysis (Xu et al., 2014; Li et al., 2015). Similarly, we established two transgenic silkworm lines to target BmJHE in the current study. One line expressing Cas9 under the control of the ubiquitous IE1 promoter (IE1-Cas9) and another line expressing sgRNAs targeting BmJHE driven by the silkworm U6 promoter (U6-sgRNAs) were crossed with each other, and somatic mutagenesis in BmJHE locus was expected in the F1 hybrid of the IE1-Cas9:U6-sgRNAs ( $\triangle J H E)$ offspring. Genomic DNA was extracted from ten randomly selected $\triangle J H E$ larvae, and the genomic fragments spanning both target sites were PCR-amplified and sequenced. As a result, both non-deletion and deletion mutations were detected, which demonstrates that this somatic mutagenesis system was effective (Fig. 2). To test whether somatic JHE mutagenesis could cause JHE inactivation, the JHE activity in the haemolymph was examined at day four of the fifth larval instar (L5D4). The result showed that JHE activity had declined sharply in the $\triangle J H E$ animals, which suggests that the biological role of JHE was 
inhibited after mutagenesis (Fig. 3).

\subsection{Extension of the larval period in $\triangle J H E$ animals}

A previous study showed that the transgenic overexpression of $B m J H E$ by the binary Gal4/UAS system shortens the duration of larval periods and induces precocious metamorphosis in B. mori (Tan et al., 2005). To investigate whether $B m J H E$ depletion has the opposite effect on the duration of larval periods, we compared the developmental time of larval stages among the $\triangle J H E$ line and three other lines (WT, IE1-Cas9 and U6-sgRNAs). No significant variation was observed in developmental timing during the early larval stages (from first to third larval instar) between $\triangle J H E$ and other control animals (data not shown). Thereafter, the developmental time of the fourth and fifth larval instars increased $\sim 1$ day (Fig. $4 \mathrm{a}$ and b). The total developmental time of the larval stages from egg deposition to wandering increased from 19.5 days to 21.5 days in mutant animals (Fig. 4c). By contrast, developmental time from pupation to eclosion showed no difference between mutant and control animals (data not shown).

Extension of the feeding stages, especially the fourth and fifth larval stages, resulted in increased biological mass. An increase in body size can be caused by either an increase in the duration of feeding stage or an enhanced rate of growth. We therefore investigated the rate of growth of $\triangle J H E$ animals after the fourth ecdysis. Both control and $\triangle J H E$ animals gained mass at approximately the same rate (Fig. 4d), but the mutants continued to feed and accumulated mass due to longer developmental time. Both the cocoons and pupae of $\triangle J H E$ animals were larger than in WT animals (Fig. 5). Female mutant whole cocoons were $47 \%$ heavier than WT and male mutant whole cocoons were $58 \%$ heavier than WT (Fig. 5e).

\subsection{RNA-seq and q-RT-PCR analysis in $\triangle J H E$ animals}

To explore the molecular mechanisms underlying how the depletion of JHE 
induced the above phenotypes, total RNA from fat bodies of WT and $\triangle J H E$ L5D4 larvae was extracted and subjected to RNA-seq analysis. Among the 784 differentially expressed genes (DEGs) identified, 287 genes were upregulated, and 497 were downregulated (Fig. 6a) in $\triangle J H E$ animals. KEEG enrichment analysis revealed that protein processing in the endoplasmic reticulum was the most affected pathway. In addition, amino acid metabolic pathways, such as the metabolism of tyrosine, alanine, aspartate and glutamate, cysteine and methionine, phenylalanine, arginine and proline, and the biosynthesis of tyrosine and tryptophan changed significantly between the WT and $\triangle J H E$ animals (Fig. 6b). We further investigated the expression levels of protein-processing genes in the endoplasmic reticulum pathway by using q-RT-PCR. Two genes of UDP-glucose-glycoprotein glucosyltransferase (Ugt) and Manl, were upregulated 3.4- and 2.5-fold, respectively, in $\triangle J H E$ animals (Fig. 7).

The insulin/TOR-signalling network has been demonstrated to play a central role in regulating cell growth and metabolism in insects. Importantly, the insulin/TOR pathway is modulated by circulating hormones, especially those involved in developmental transition (Mirth et al., 2014; Li et al., 2015). The activation of the insulin/TOR pathway promotes cap-dependent protein synthesis and cell growth through the phosphorylation of the ribosomal protein S6 kinases (S6K) and eukaryotic initiation factor 4E-1 (eIF4E-1), at least in part. Although the RNA-Seq results showed no significant variations in the insulin/TOR pathway, we hypothesized that the depletion of JHE might affect the development timing and body size of larvae via the insulin/TOR pathway. We compared the expression of known components of the insulin/TOR pathway in $\triangle J H E$ animals with controls; the q-RT-PCR results showed that three positively regulated genes, phosphoinositide 3-kinase (PI3K), AKT and S6K, were upregulated 1.4-, 2.6-, and 2.4-fold, respectively (Fig. 7). Interestingly, the transcription level of eIF4E-1 was upregulated up to 113 -fold in $\triangle J H E$ animals (Fig. 8). By contrast, the mRNA levels of two negatively regulated genes, tuberous sclerosis 2 (TSC2) and eIF4E-binding proteins (4E-BP), decreased to $38.0 \%$ and 
$48.1 \%$ of their levels in control animals (Fig. 7).

We also investigated expression variation of those genes in $\mathrm{JH}$ signalling pathway by q-RT-PCR. In $\triangle J H E$ animals, $J H E$ mRNA levels decreased to $40.2 \%$ comparing to WT animals (Fig. 7). On the contrary, expression levels of JHEH and JHDK, two JH degradation enzymes, were upregulated 1.8- and 4.1-fold, respectively, in $\triangle J H E$ animals (Fig. 7). We also detected the transcript level of the JH-inducible transcription factor, Krüppel homolog 1 (Kr-h1), as the indicator of JH titer. The mRNA level of $K r-h 1-\beta$ was upregulated 3.2-fold. However, the transcript level of $K r-h 1-\alpha$ did not change significantly (Fig. 7).

\section{Discussion}

In the current study, we exploited the transgenic CRISPR/Cas9 system to elucidate the biological function of JHE in B. mori. As the primary JH-specific degradation enzyme, JHE plays key roles in regulating circular JH titres in insects. The overexpression of JHE could induce precocious metamorphosis in B. mori (Tan et al., 2005), whereas the application of a JHE antagonist could extend larval duration in Manduca sexta (Akai and Kobayashi, 1971; Akai et al., 1973; Abdel-Aal and Hammock, 1986). Our data present the first example of JHE gene elimination in insects and provide a promising approach for exploiting $\mathrm{JH}$ action, especially in lepidopteran insects.

Somatic mutagenesis of $B m J H E$ and the subsequently reduced JHE activity extended the larval period and increased biological mass. A putative decline in the $\mathrm{JH}$ degradation rate due to $B m J H E$ depletion is likely responsible for this phenotype, although the depletion of JHE trigged the compensatory pathway in which expression of JHEH and JHDK were upregulated. Similarly, the application of JH or a $\mathrm{JH}$ analogue delayed metamorphosis, resulting in larger body size in B. mori (Akai and Kobayashi, 1971; Akai et al., 1973). Although the transgenic CRISPR/Cas9 system induces mutagenesis from the embryonic stage, no significant differences were 
observed between the $\triangle J H E$ and control animals before the third larval instar. This phenotype was similar to the overexpression of BmJHE (Tan et al., 2005) or JH-deficient mutants in B.mori (Daimon et al., 2012), which indicates that JH is critical to the late larval stages (fourth and fifth instar stages) rather than the earth larval stages (from the first to third larval instar stage). In the last instar of the silkworm, the CA stops synthesize $\mathrm{JH}$ by stopping the expression of $\mathrm{JH}$ biosynthesis genes including JHAMT (Shinoda and Itoyama, 2003; Kinjoh et al., 2007). At the same time, JHE expression was elevated to remove abundant JH (Hirai et al., 2002). Our work clearly showed that JHE is indeed important for the control of developmental timing prior to pupation probably by accelerating the clearance of $\mathrm{JH}$ in the hemolymph. In Drosophila, JH regulates body size by controlling the growth rate, which is insulin pathway-dependent (Mirth et al., 2014). In M. sexta, JH regulates body size by controlling the duration of growth (Mirth and Riddiford, 2007). The cessation of growth in $M$. sexta is associated with the attainment of critical weight in the fifth larval instar followed by a terminal growth period, and applying JH to larvae extended the larval period (Mirth and Riddiford, 2007; Nijhout and Williams, 1974). Recently, the insulin/TOR pathway was demonstrated to be involved in the determination of the final body size in Drosophila and M. sexta (Hatem et al., 2015). Our data suggested that the insulin/TOR pathway was activated in $\triangle J H E$ animals. This is probably due to abundant $\mathrm{JH}$ in $\triangle J H E$ animals could modulate the insulin/TOR pathway positively (Mirth and Shingleton, 2014). As a result, the transcription level of $e I F 4 E-1$ was upregulated by up to 113 -fold. This gene is a downstream component of the insulin/TOR pathway and plays a critical role in the regulation of translation initiation (Wilhelm and Smibert, 2005), which is the rate-limiting process for protein biosynthesis (Kong and Lasko, 2012).

The silkworm is not only a lepidopteran model insect but also an economically important insect. Our data showed that the cocoon yield increased by $\sim 30 \%$ due to JHE depletion (Fig 5 and 6) without the appearance of any deleterious phenotypes. Because this study is an important proof-of-principle carried out in a strain suitable 
only for research (Nistari), its economic characteristics (silk quantity and quality) are inferior to those of pure commercial strains. Either outcrossing our transgenic line to commercial strains or directly performing JHE depletion through the transgenic CRISPR/Cas9 system in commercial strains will greatly facilitate silk production in the future.

\section{Acknowledgements}

This work was supported by grants from the Strategic Priority Research Program of Chinese Academy of Sciences (XDB11010500 and XDA04020202) and the National Science Foundation of China (31420103918 and 31530072).

\section{References}

Abdel-Aal Y.A., Hammock B.D., 1986. Transition state analogs as ligands for affinity purification of juvenile hormone esterase. Science. 233, 1073-1076.

Akai, H. and M. Kobayashi., 1971. Induction of prolonged larval instar by juvenile hormone in Bombyx mori (Lepidoptera: Bombycidae). Appl. Entomol. Zool. 6, 138-139.

Akai, H., Kiguchi, K. and K. Mori., 1973. The influence of juvenile hormone on the growth and metamorphosis of Bombyx larvae. Bull. Seric. Exp. Stn. 25, 287-305.

Bellés, X., Martín, D., Piulachs, M.D., 2005. The mevalonate pathway and the synthesis of juvenile hormone in insects. Annu Rev Entomol. 50, 181-199.

Conesa A., Götz S., García-Gómez J.M., Terol J., Talón M., Robles M., 2005. Blast2GO: a universal tool for annotation, visualization and analysis in functional genomics research. Bioinformatics. 21: 3674-3676.

Daimon, T., Kozaki, T., Niwa, R., Kobayashi, I., Furuta, K., Namiki, T., Uchino, K., Banno, Y., Katsuma, S., Tamura, T., Mita, K., Sezutsu, H., Nakayama, M., Itoyama, K., Shimada, T., Shinoda, T., 2012. Precocious metamorphosis in the juvenile hormone-deficient mutant of the silkworm, Bombyx mori. PLoS Genet. 8, e1002486. 
Gäde, G., Hoffmann, K.H., Spring, J.H., 1997. Hormonal regulation in insects: facts, gaps, and future directions. Physiol Rev. 77, 963-1032.

Hammock, B.D., Bonning, B.C., Possee, R.D., Hanzlik, T.N., Maeda, S., 1990. Expression and effects of the juvenile hormone esterase in a baculovirus vector. Nature. 344, 458-461.

Hatem, N.E., Wang, Z., Nave, K.B., Koyama, T., Suzuki, Y., 2015. The role of juvenile hormone and insulin/TOR signaling in the growth of Manduca sexta. BMC Biol. 13, 1-12.

Hirai, M., Kamimura, M., Kikuchi, K., Yasukochi, Y., Kiuchi, M., Shinoda, T., Shiotsuki, T., 2002. cDNA cloning and characterization of Bombyx mori juvenile hormone esterase: an inducible gene by the imidazole insect growth regulator KK-42. Insect Biochem.Mol. Biol. 32, 627-635.

Hinton, A.C., Hammock, B.D., 2003a. In vitro expression and biochemical characterization of juvenile hormone esterase from Manduca sexta. Insect Biochem. Mol. Biol. 33, 317-329.

Hinton, A.C., Hammock, B.D., 2003b. Juvenile hormone esterase (JHE) from Tenebrio molitor: full-length cDNA sequence, in vitro expression, and characterization of the recombinant protein. Insect Biochem. Mol. Biol. 33, $477-487$.

Hwang, W.Y., Fu, Y, Reyon, D., Maeder, M.L., Tsai, S.Q., Sander, J.D., Peterson, R.T., Yeh, J.R., Joung, J.K., 2013. Efficient genome editing in zebrafish using a CRISPR-Cas system. Nat Biotechnol. 31, 227-229.

Jindra, M., Palli, S.R., Riddiford, L.M., 2013. The juvenile hormone signaling pathway in insect development. Annu Rev Entomol. 58, 181-204.

Kamita, S.G., Hammock, B.D., 2010. Juvenile hormone esterase: biochemistry and structure. J Pestic Sci. 35, 265-274.

Kanehisa M., Araki M., Goto S., Hattori M., Hirakawa M., Itoh M., Katayama T., Kawashima S., Okuda S., Tokimatsu T., Yamanishi Y., 2008. KEGG for linking genomes to life and the environment. Nucleic Acids Res. 36, 480-484.

Kinjoh T., Kaneko Y., Itoyama K., Mita K., Hiruma K., Shinoda T., 2007. Control of 
juvenile hormone biosynthesis in Bombyx mori: cloning of the enzymes in the mevalonate pathway and assessment of their developmental expression in the corpora allata. Insect Biochem Mol Biol. 37, 808-818.

Kong J., Lasko P., 2012. Translational control in cellular and developmental processes. Nat Rev Genet. 13, 383-394.

Li, S., Zhang, Q.R., Xu, W.H., Schooley, D.A., 2005. Juvenile hormone diol kinase, a calcium-binding protein with kinase activity, from the silkworm, Bombyx mori. Insect Biochem Mol Biol. 35, 1235-1248.

Li, Z., You, L., Zeng, B., Ling, L., Xu, J., Chen, X., Zhang, Z., Palli, S.R., Huang, Y., Tan, A., 2015. Ectopic expression of ecdysone oxidase impairs tissue degeneration in Bombyx mori. Proc Biol Sci. 282, 3238-3248.

McCutchen B.F., Szekacs A., Huang T.L., Shiotsuki T., Hammock B.D., 1995. Characterization of a spectrophotometric assay for juvenile hormone esterase. Insect Biochem Mol Biol. 25, 119-126.

Mirth, C.K., Riddiford, L.M., 2007. Size assessment and growth control: how adult size is determined in insects. Bioessays. 29, 344-355.

Mirth C.K., Shingleton A.W., 2014. The roles of juvenile hormone, insulin/target of rapamycin, and ecydsone signaling in regulating body size in Drosophila. Commun Integr Biol. 7, 1-3.

Mirth C.K., Tang H.Y., Makohon-Moore S.C., Salhadar S., Gokhale R.H., Warner R.D., Koyama T., Riddiford L.M., Shingleton A.W., 2014. Juvenile hormone regulates body size and perturbs insulin signaling in Drosophila. Proc Natl Acad Sci U S A. 111, 7018-7023.

Nijhout, H.F., Williams, C.M., 1974. Control of moulting and metamorphosis in the tobacco hornworm, Manduca sexta $(L$.$) : cessation of juvenile hormone secretion$ as a trigger for pupation. J Exp Biol. 61, 493-501.

Niwa, R., Niwa, Y.S., 2014. Enzymes for ecdysteroid biosynthesis: their biological functions in insects and beyond. Biosci Biotechnol Biochem. 78, 1283-1292.

Riddiford, L.M., 1996. Juvenile hormone: the status of its "status quo" action. Arch Insect Biochem Physiol. 32, 271-286. 
Riddiford, L.M., Truman, J.W., Mirth, C.K., Shen, Y.C., 2010. A role for juvenile hormone in the prepupal development of Drosophila melanogaster. Development $137,1117-1126$

Riddiford, L.M., 2012. How does juvenile hormone control insect metamorphosis and reproduction? Gen Comp Endocrinol. 179, 477-484.

Seino, A., Ogura, T., Tsubota, T., Shimomura, M., Nakakura, T., Tan, A., Mita, K., Shinoda, T., Nakagawa, Y., Shiotsuki, T., 2010. Characterization of juvenile hormone epoxide hydrolase and related genes in the larval development of the silkworm Bombyx mori. Biosci Biotechnol Biochem. 74, 1421-1429.

Shinoda T., Itoyama K., 2003. Juvenile hormone acid methyltransferase: a key regulatory enzyme for insect metamorphosis. Proc Natl Acad Sci U S A. 100, 11986-11991.

Sparks, T.C., Hammock, B.D., 1980. Comparative inhibition of the juvenile hormone esterases from Trichoplusia ni, Tenebrio molitor, and Musca domestica. Pestic. Biochem. Physiol. 14, 290-302.

Tan, A., Tanaka, H., Tamura, T., Shiotsuki, T., 2005. Precocious metamorphosis in transgenic silkworms overexpressing juvenile hormone esterase. Proc Natl Acad Sci U S A. 102, 11751-11756.

Tan, A., Fu, G., Jin, L., Guo, Q., Li, Z., Niu, B., Meng, Z., Morrison, N.I., Alphey, L., Huang, Y., 2013. Transgene-based, female-specific lethality system for genetic sexing of the silkworm, Bombyx mori. Proc Natl Acad Sci U S A. 110, 6766-6670.

Tarazona S., García-Alcalde F., Dopazo J., Ferrer A., Conesa A., 2011. Differential expression in RNA-seq: a matter of depth. Genome Res. 21, 2213-2223.

Truman, J.W., Riddiford, L.M., 2007. The morphostatic actions of juvenile hormone. Insect Biochem Mol Biol. 37, 761-770.

Wilhelm J.E., Smibert C.A., 2005. Mechanisms of translational regulation in Drosophila. Biol Cell. 97, 235-252.

Xu J., Wang Y., Li Z., Ling L., Zeng B., James A.A., Tan A., Huang Y., 2014. Transcription activator-like effector nuclease (TALEN)-mediated female-specific sterility in the silkworm, Bombyx mori. Insect Mol Biol. 23, 800-807. 


\section{Figures legends}

Fig. 1. Schematic diagram of $B m J H E$ sgRNA targeting sites and transgenic plasmids. (a) Schematic diagram of sgRNA-targeting sites. The boxes indicate the six exons of BmJHE, and the black line represents the gene locus. The sgRNA-targeting sites, S1 and S2, are located on the sense strand of the exon-3. Primers of TS-F and TS-R were used to anneal the upstream and downstream regions of the targeting sites, respectively. The sgRNA targeting sequence is in black, and the protospacer adjacent motif (PAM) sequence is in red. (b) Schematics of the pBac[U6-BmJHEsgRNA-IE1-DsRed2] (U6-sgRNAs) vector. BmJHE-specific sgRNA expression was driven by the U6 promoter. (c) Schematics of the pBac[IE1-Cas9-IE1-EGFP] (IE1-Cas9) vector. The Cas9 expression was driven by the IE1 promoter.

Fig. 2. Genomic mutagenesis induced by the transgenic CRISPR/Cas9 system. Various deletion or non-deletion mutagenesis were detected in heterozygous IE1-Cas9:U6-sgRNAs ( $\triangle J H E)$ offspring. In non-deletion events, few nucleotides were removed at a single target site. In deletion events, the fragments spanning the two sites were deleted. The numbers in brackets in the middle of each sequence refer to the 64-bp interspace fragment that was found between the targeting sites. The red sequence indicates the PAM sequence. The genotypes represents the various genotypes of three $\triangle J H E$ animals.

Fig. 3. The activity of JHE declined sharply in $\triangle J H E$ animals. JHE activity in the haemolymph was measured with the spectrophotometric JH-mimic substrate methyl heptylthioacetothioate. The data shown are mean \pm SEM $(n=3)$. The asterisks indicate the significant differences with a two-tailed t-test: $* * * \mathrm{P}<0.0001$. 
Fig. 4. Loss of $B m J H E$ function resulted in extended larval periods and increased larval weight. The percentages of larvae of the indicated genotype that ecdysed to the (a) the fourth larval instar, (b) the fifth larval instar, and (c) wandering stage are plotted relative to the time in days after egg hatching. (d) Plot of the weight at a given time after L4 ecdysis for the indicated genotype. Black is $+/+$, red is U6-sgRNAs/+, dark green is $I E 1-C a s 9 /+$, and purple is $\triangle J H E$ animals. The data shown are mean \pm SEM $(n=8-10)$. The asterisks indicate the significant differences with a two-tailed t-test: $* \mathrm{P}<0.05 ; * * \mathrm{P}<0.01 ; * * * \mathrm{P}<0.0001$

Fig. 5.Giant pupae and cocoons in $\triangle J H E$ animals. (a, c) Pupae of $\triangle J H E$ or from

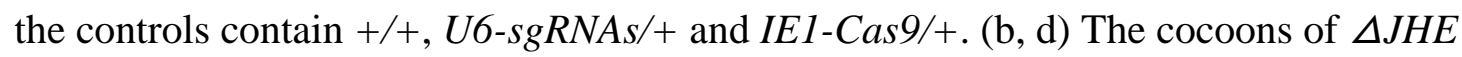
or from the controls contain wild-type, U6-sgRNAs/+ and IE1-Cas9/+. (e) The economic traits of the indicated phenotype. The data shown are mean \pm SEM $(n=30)$. The asterisks indicate the significant differences with a two-tailed t-test: $* \mathrm{P}<0.05$; $* * \mathrm{P}<0.01$

Fig. 6. RNA-Seq analysis showed that protein processing and amino acid pathways were affected in $\boldsymbol{\Delta J H E}$ animals. (a) The significantly enriched gene ontology terms for biological processes, cellular components and molecular functions of the differentially expressed genes (DEGs). (b) The top ten enriched KEGG pathways.

Fig. 7. Relative gene expression ratios determined by q-RT-PCR in $\Delta J H E$ animals. The expression levels of $\mathrm{JH}$ metabolic genes $J H E, J H E H$ and $J H D K$, the endoplasmic reticulum pathway-associated genes UDP-glucose-glycoprotein glucosyltransferase $(\underline{U g t})$ and Manl, the insulin/TOR pathway-associated genes phosphoinositide 3-kinase (PI3K), AKT, ribosomal protein S6 kinases (S6K), eukaryotic initiation factor 4E-1 (eIF4E-1), tuberous sclerosis 2 (TSC2) and 
eIF4E-binding proteins (4E-BP), and the JH response gene Krüppel homolog $1(\mathrm{Kr}-\mathrm{hl})$ were investigated. Representative data from at least three independent experiments are shown. The data shown are mean \pm SEM $(n=3)$. The asterisks indicate the significant differences with a two-tailed t-test: $* \mathrm{P}<0.05 ; * * \mathrm{P}<0.01 ; * * * \mathrm{P}<0.0001$. 


\section{$B m \boldsymbol{H E}$}

a

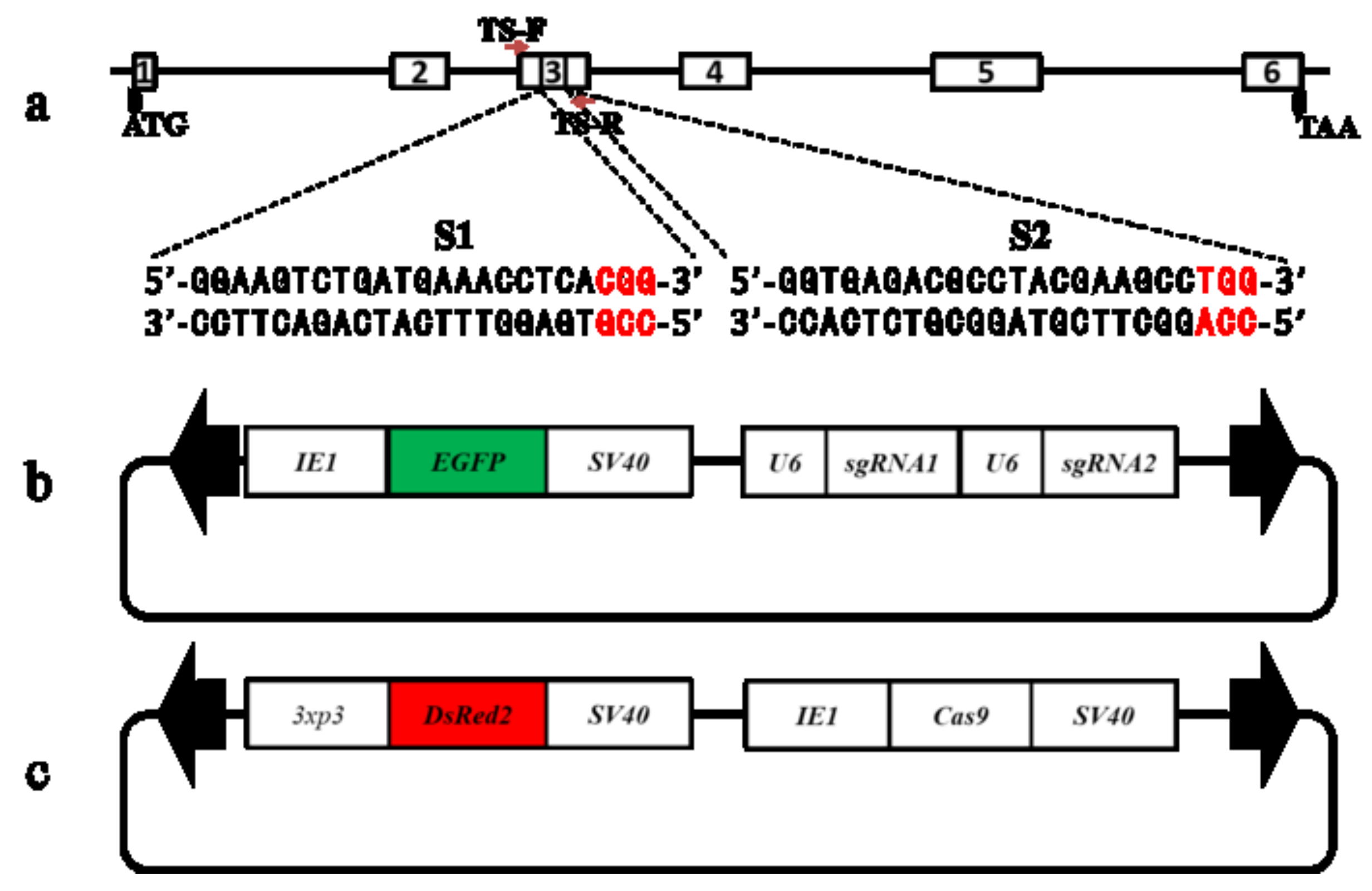

b
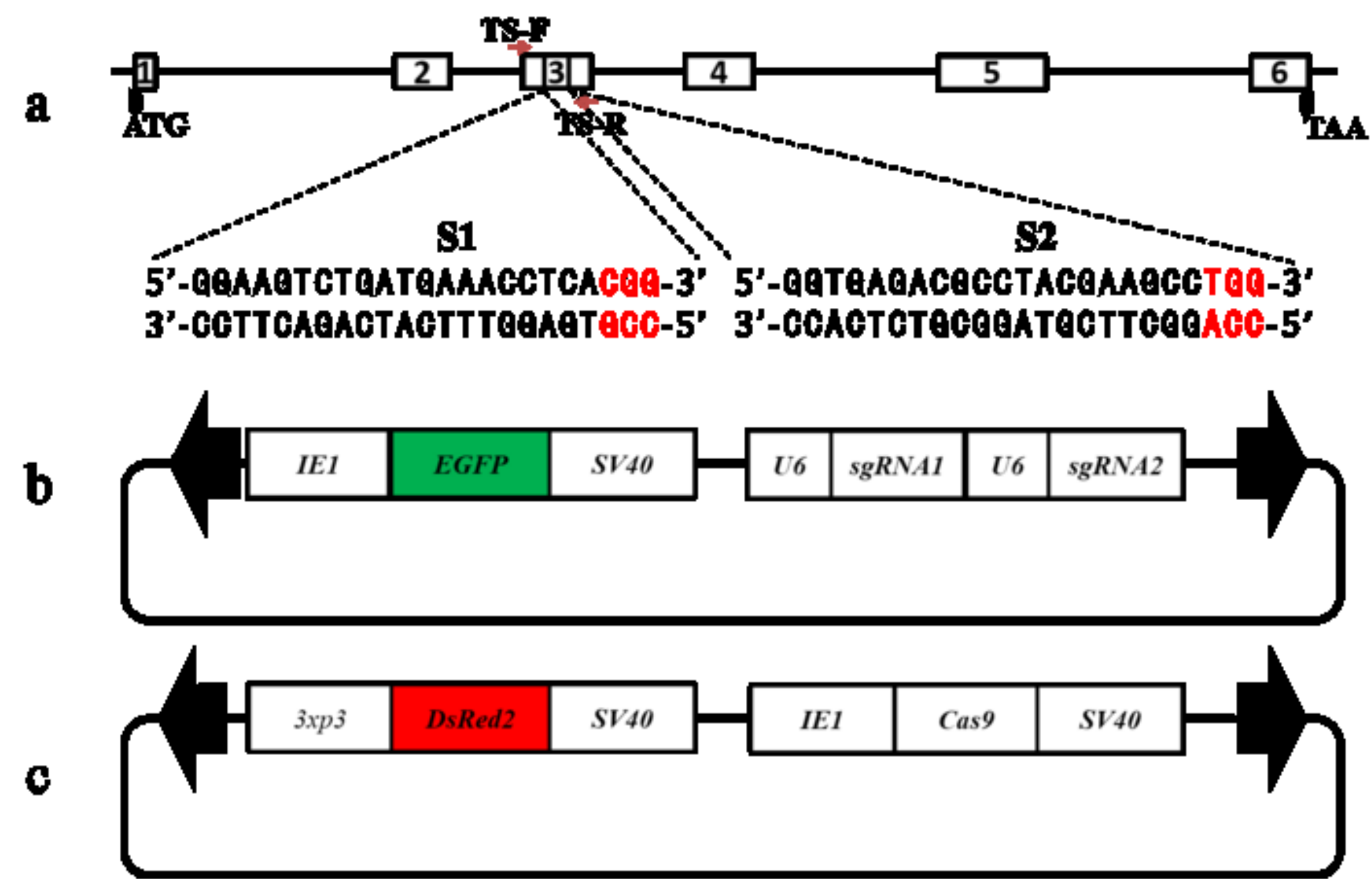
non-deletion events

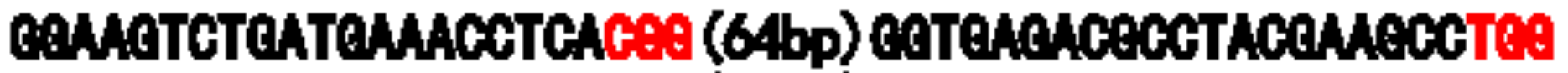

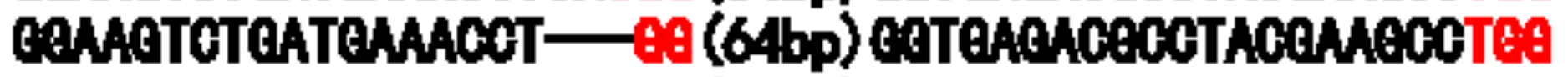
GQNACTOTOATONAA - CACQO (64bp) OOTEACNCECOTACCAACCOTEO

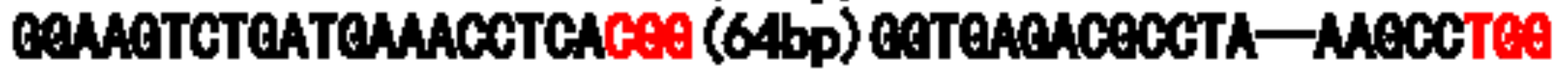
CQAACTCTCA-ACOTCACOA (64bp) OOTEACACECOT ACCANEO-O

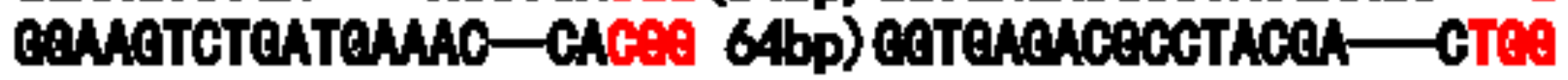

deletion events

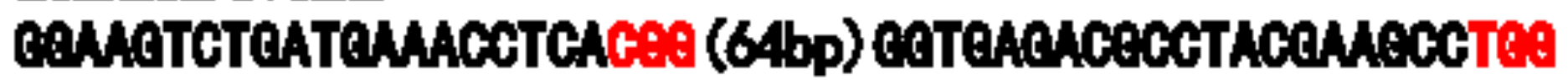
GRAGTCTCATCAAACOTOGQAATCTOATOA —- - COTEO GENAGTOTGA 

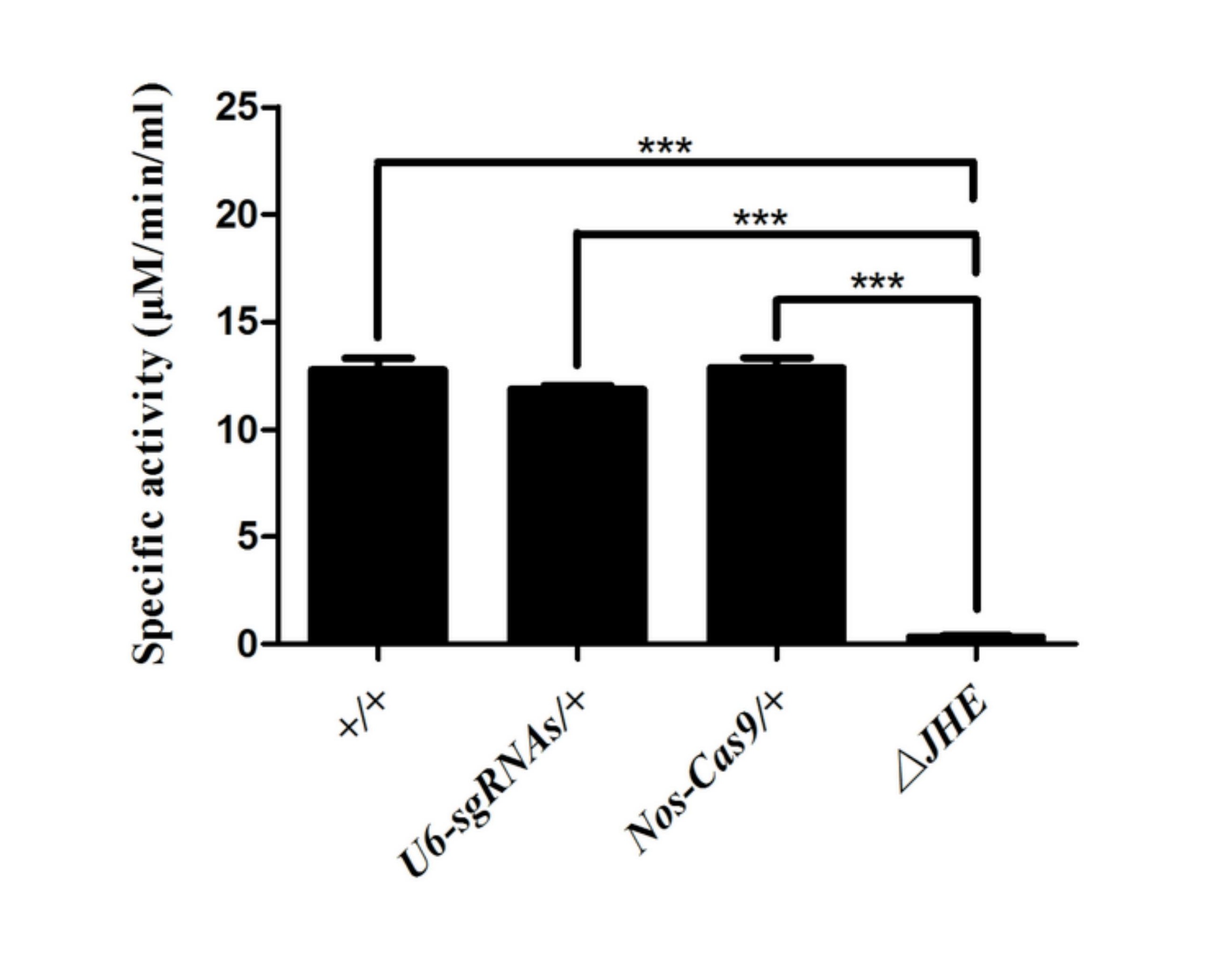

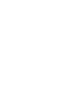


a

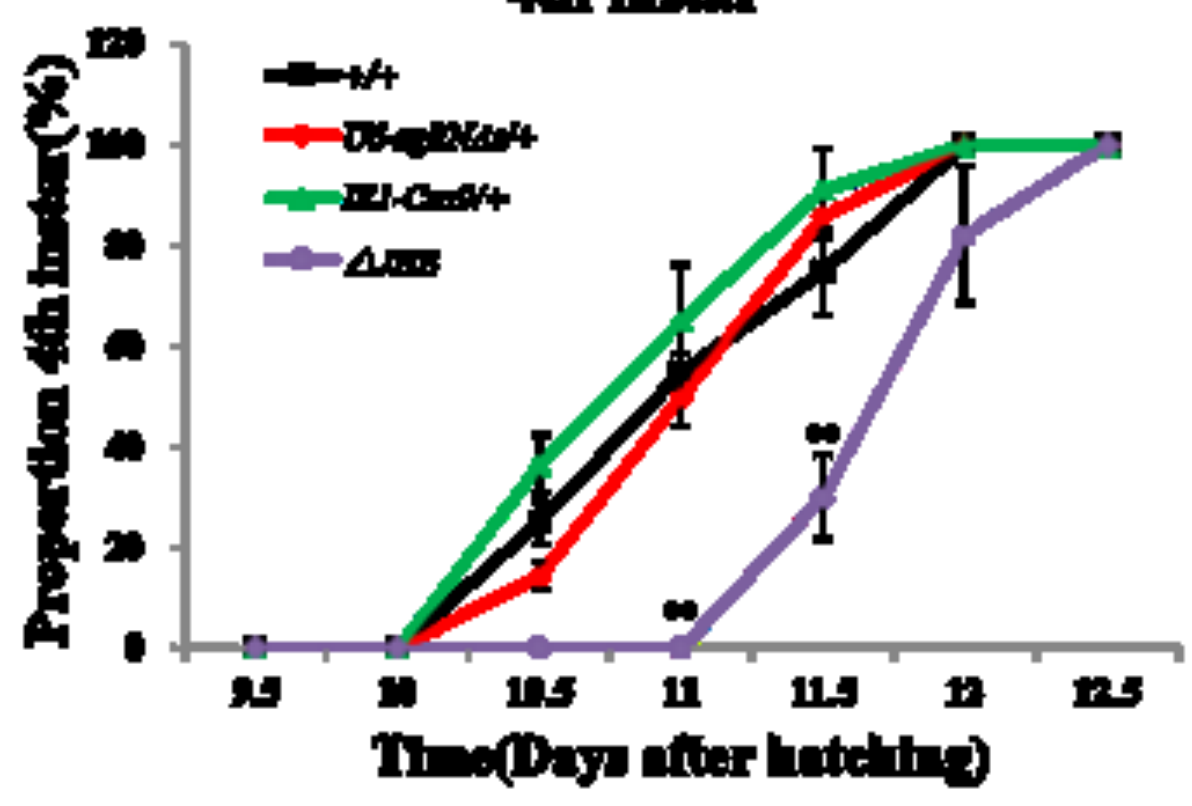

c

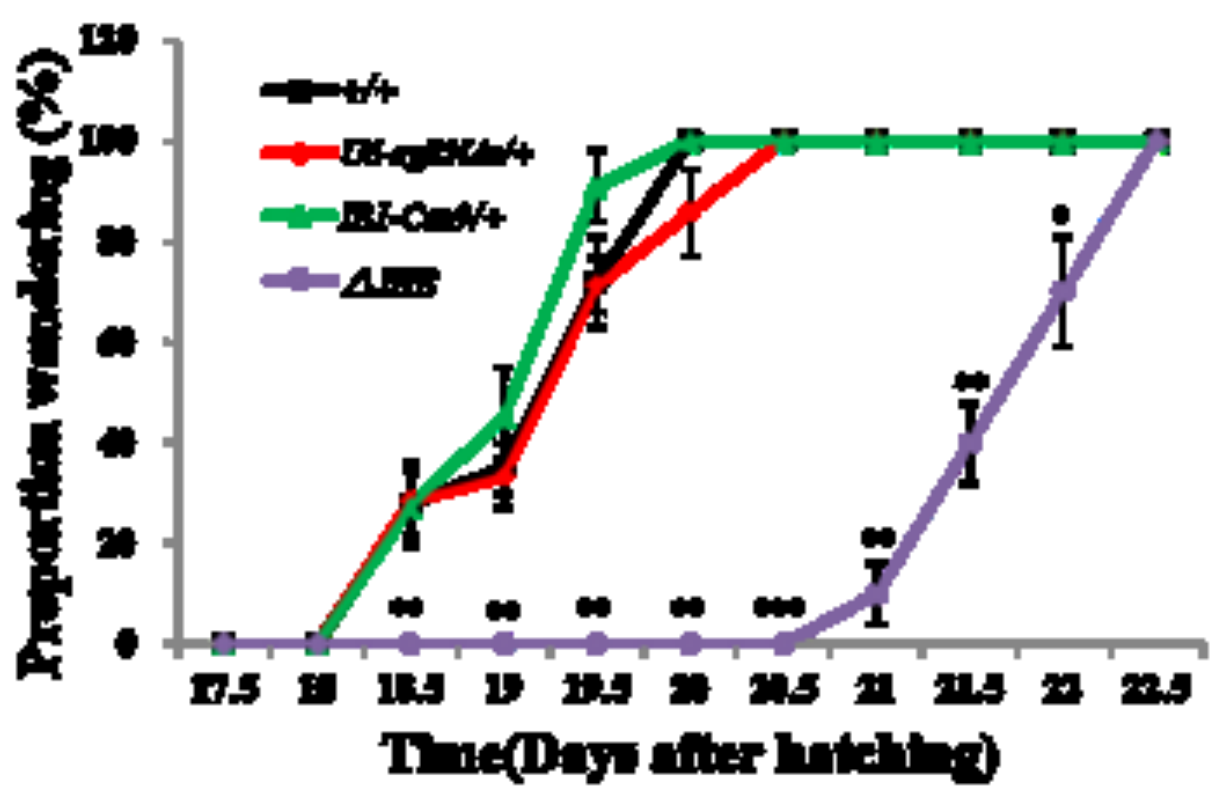

b

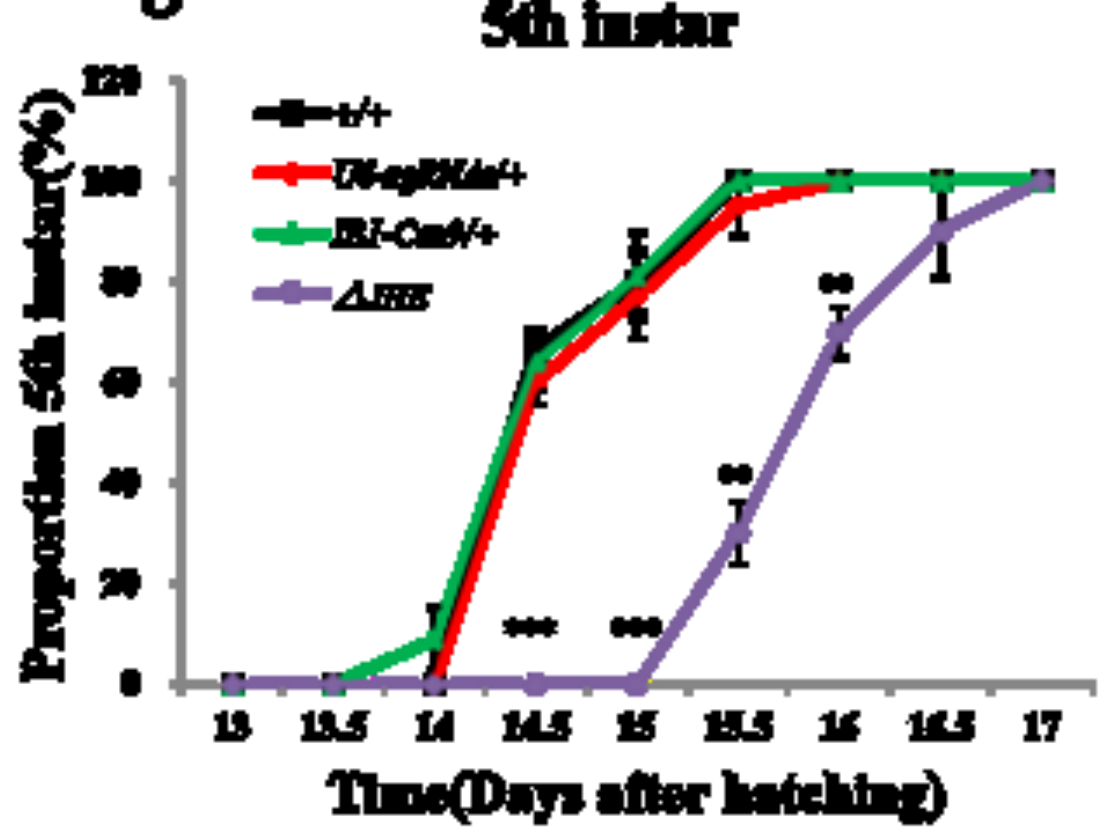

d

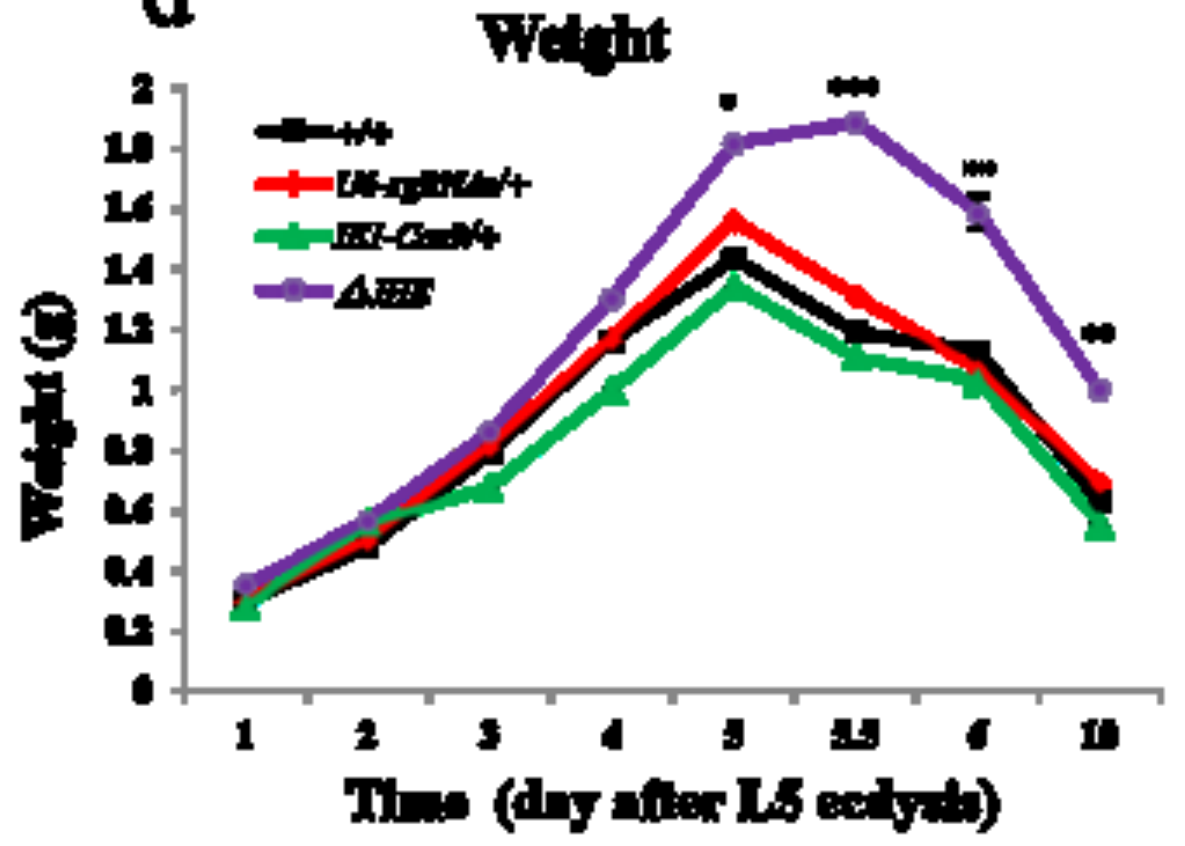



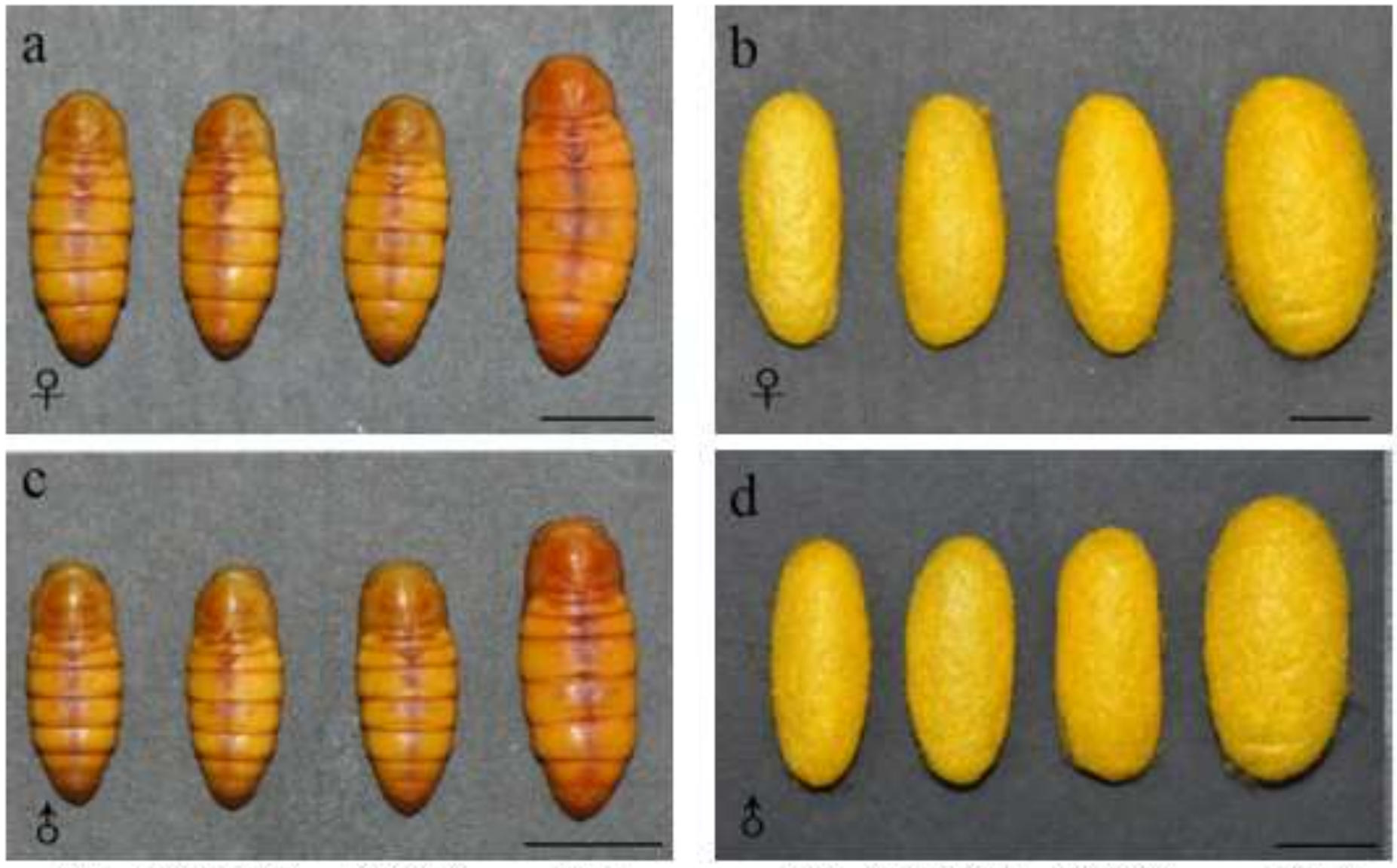

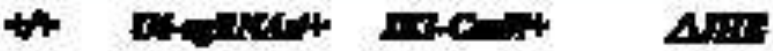

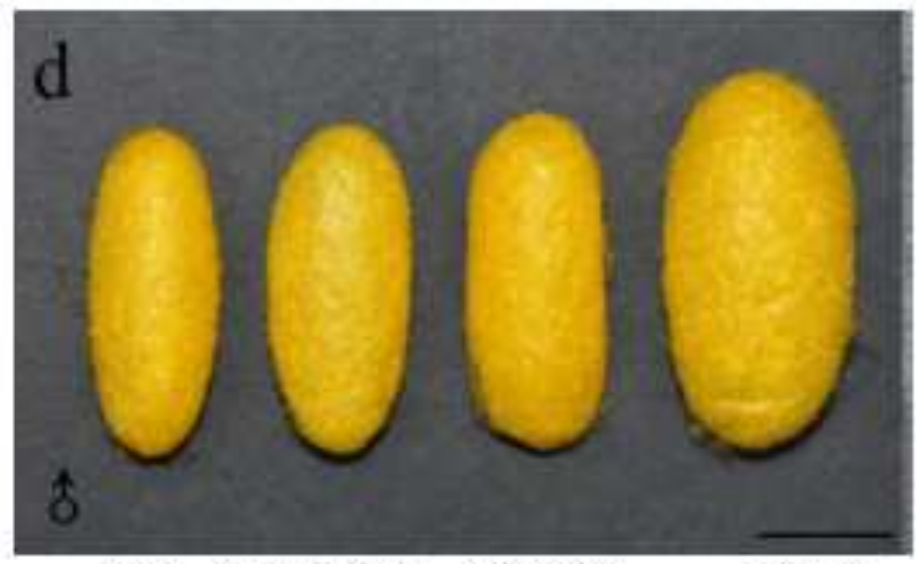

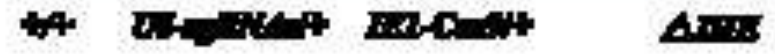

e

FEMALE

MALE

\begin{tabular}{cccccc}
\hline $\begin{array}{c}\text { Whole cocoon } \\
\text { weight }(\mathrm{mg})\end{array}$ & $\begin{array}{c}\text { Cocoon } \\
\text { shell weight } \\
(\mathrm{mg})\end{array}$ & $\begin{array}{c}\text { Cocoon } \\
\text { shell rate }(\%)\end{array}$ & $\begin{array}{c}\text { Whole cocoon } \\
\text { weight }(\mathrm{mg})\end{array}$ & $\begin{array}{c}\text { Cocoot } \\
\text { shell weight } \\
(\mathrm{mg})\end{array}$ & $\begin{array}{c}\text { Cocoon } \\
\text { shell rate }(\%)\end{array}$ \\
$730 \pm 20$ & $70 \pm 1$ & $9.61 \pm 0.12$ & $524 \pm 16$ & $67 \pm 3$ & $12.83 \pm 0.18$ \\
$750 \pm 13$ & $77 \pm 5$ & $10.28 \pm 0.08$ & $606 \pm 9$ & $78 \pm 2$ & $12.88 \pm 0.08$ \\
$649 \pm 20$ & $69 \pm 3$ & $9.42 \pm 0.13$ & $459 \pm 19$ & $56 \pm 3$ & $12.33 \pm 0.18$ \\
$1075 \pm 23^{*}$ & $101 \pm 3^{* *}$ & $9.46 \pm 0.16$ & $830 \pm 20^{*}$ & $101 \pm 5^{* *}$ & $12.06 \pm 0.32$
\end{tabular}

$\triangle H E$

$1075 \pm 23$

$101 \pm 3$

$9.46 \pm 0.16$

$830 \pm 20$

$101 \pm 3$

$12.06 \pm 0.32$ 
a.

\begin{tabular}{lcc}
\hline \multicolumn{1}{c}{ Pathway } & Pathway ID & P-value \\
\hline Protein processing in endoplasmic reticulum & Ko04141 & $8.925193 \mathrm{e}-15$ \\
Metabolic pathways & ko01100 & $8.232471 \mathrm{e}-11$ \\
Antigen processing and presentation & ko04612 & $1.081959 \mathrm{e}-06$ \\
Lysosome & ko04142 & $1.345272 \mathrm{e}-05$ \\
Riboflavin metabolism & ko00740 & $3.747454 \mathrm{e}-05$ \\
Tyrosine metabolism & ko00350 & $5.949958 \mathrm{c}-05$ \\
Alanine, aspartate and glutamate metabolism & ko00250 & $9.123642 \mathrm{e}-05$ \\
Cysteine and methionine metabolism & ko00270 & 0.0009144373 \\
Phenylalanine, tyrosine and tryptophan biosynthesis & ko00400 & 0.001155742 \\
Arginine and proline metabolism & Ko00330 & 0.001985582 \\
\hline
\end{tabular}

b

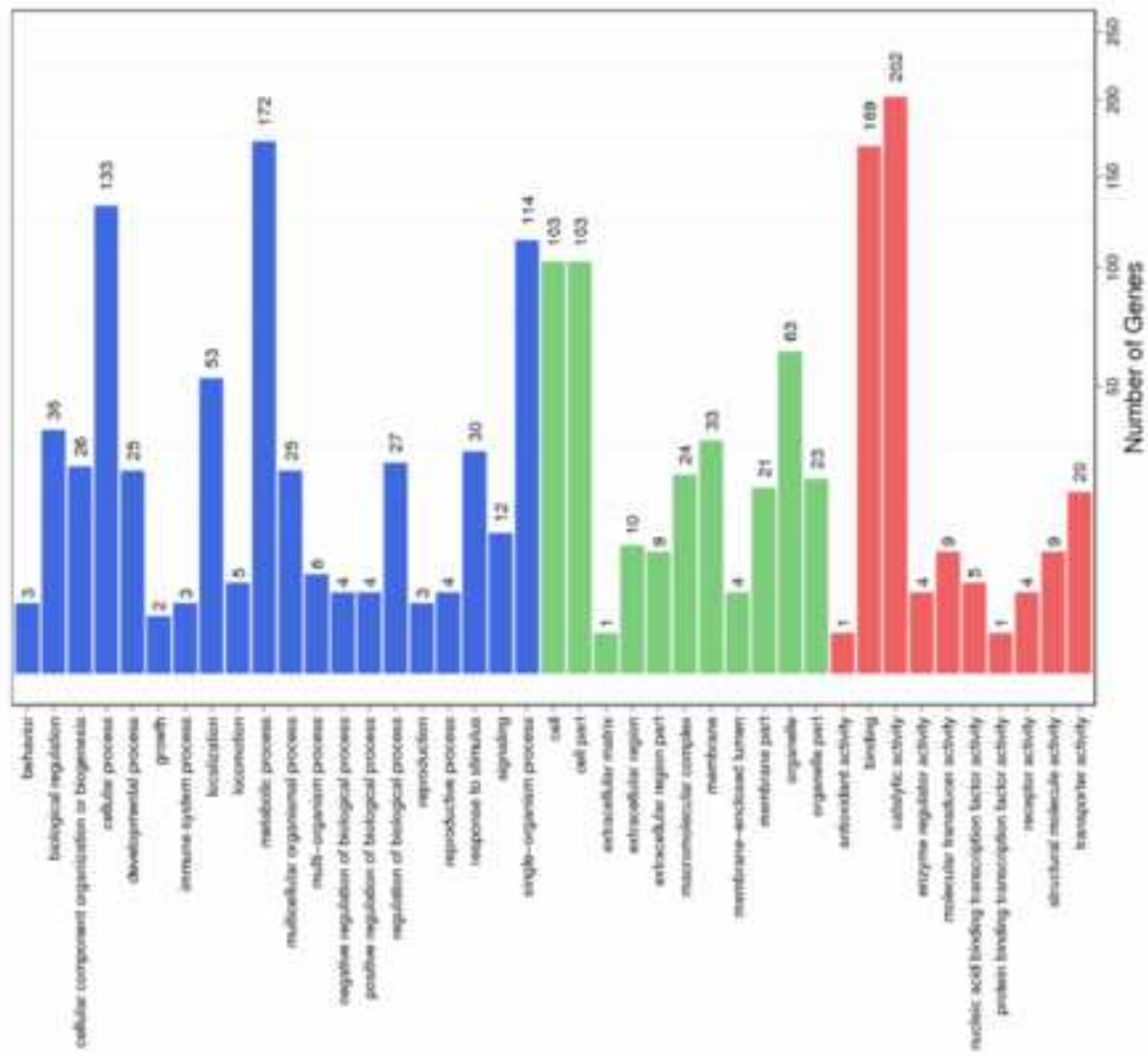




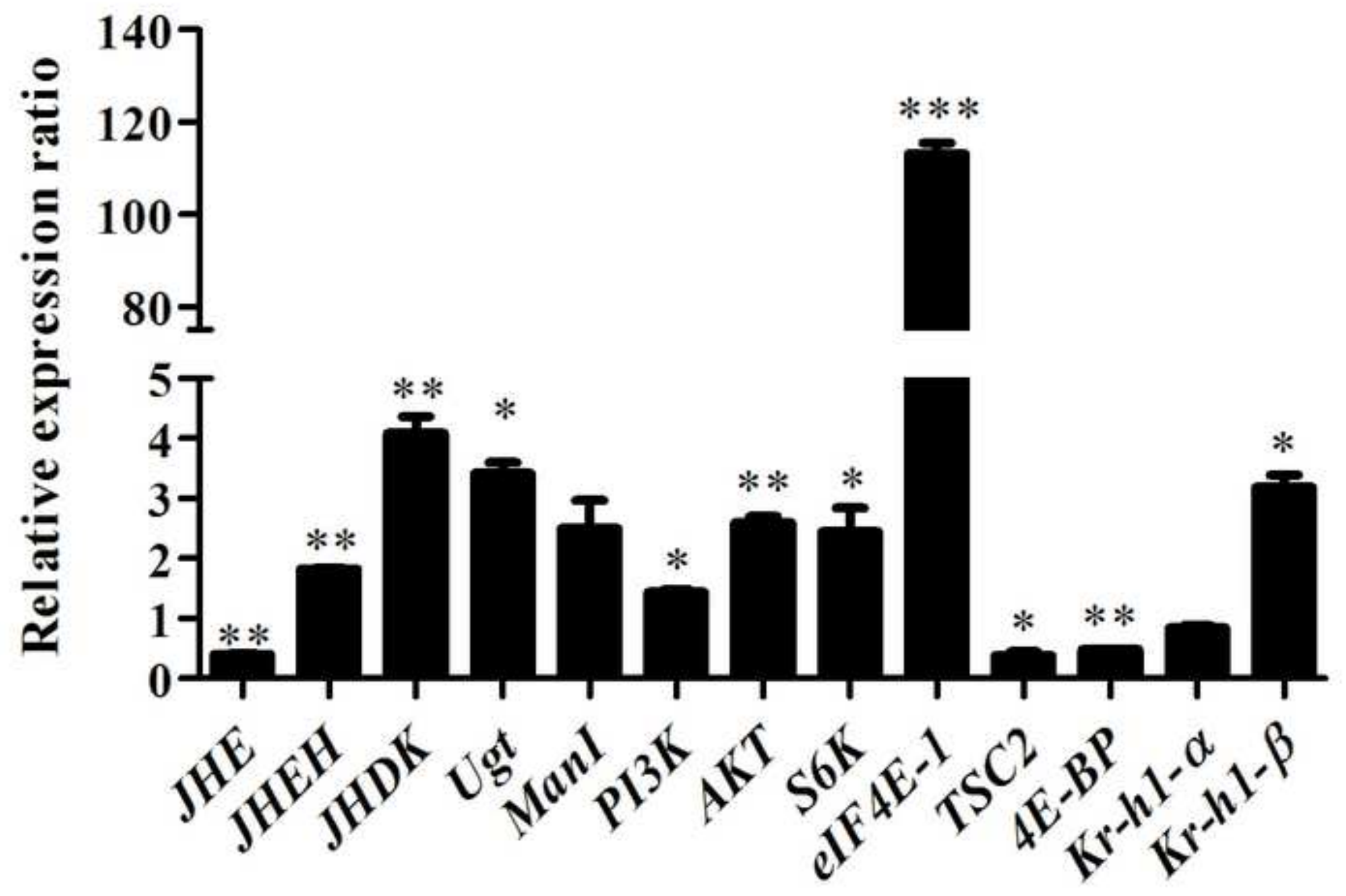



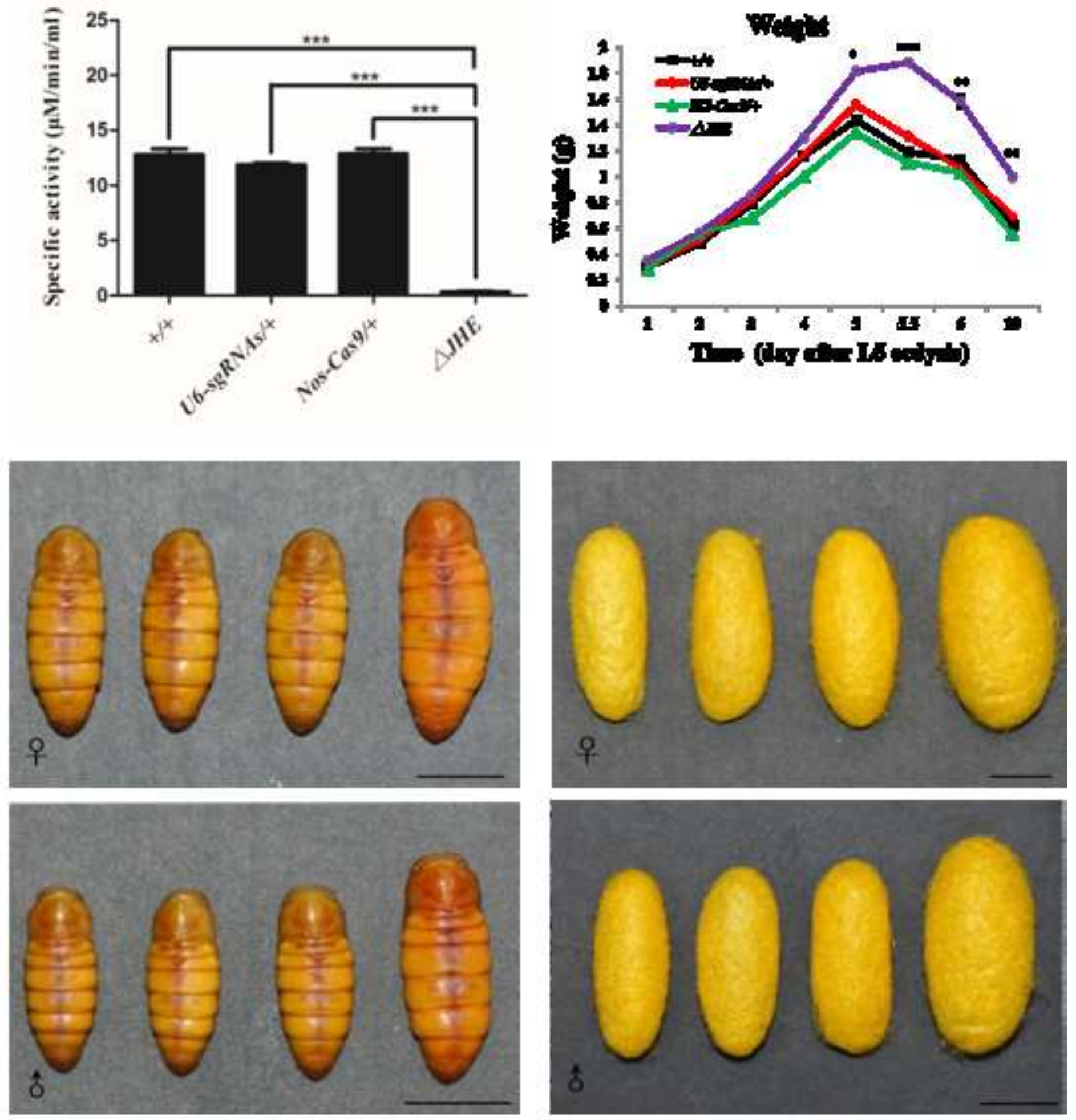

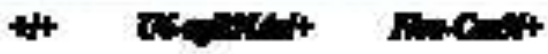
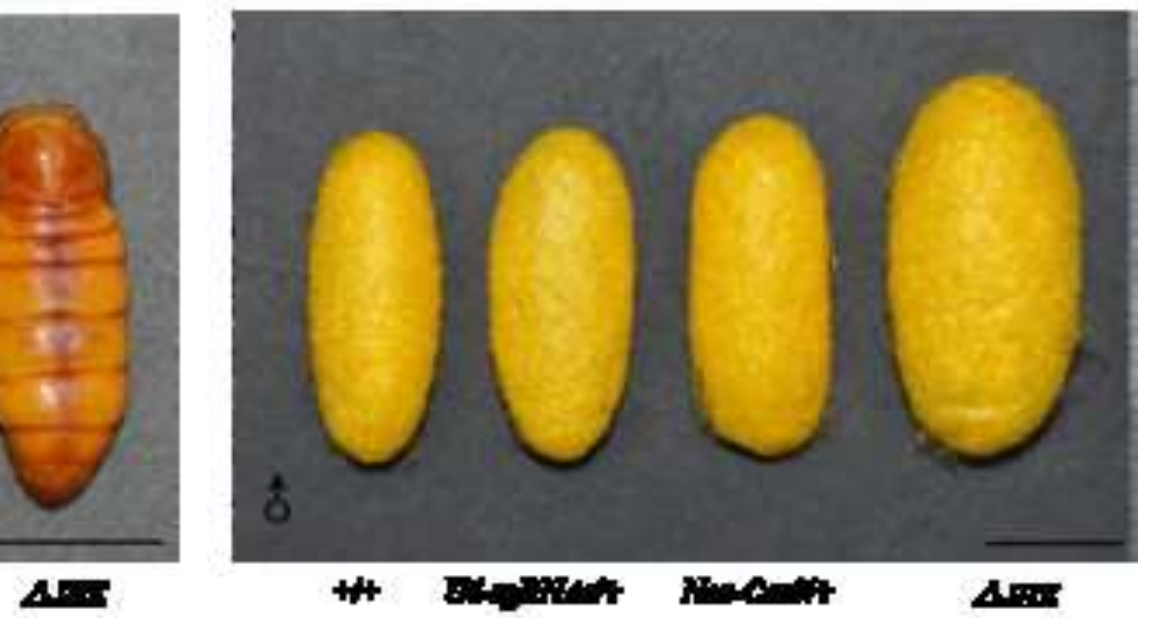